\title{
A CLASS NUMBER FORMULA OF IWASAWA'S MODULES
}

\author{
By TAKashi Fukuda
}

\section{$\S 0$. Introduction.}

Let $p$ be an odd prime number which will be fixed throughout the following. Let $k$ be a finite extension of $\boldsymbol{Q}$ and $k_{\infty}$ be the cyclotomic $\boldsymbol{Z}_{p}$-extension $k \boldsymbol{Q}_{\infty}$ of $k$, where $\boldsymbol{Q}_{\infty}$ is the unique $\boldsymbol{Z}_{p}$-extension of $\boldsymbol{Q}$ (c.f. [6]). For any $n \geqq 0$, let $k_{n}$ be the unique extension of $k$ in $k_{\infty}$ of degree $p^{n}$ over $k: k=k_{0} \subset k_{1} \subset \cdots \subset k_{\infty}$, and let $\Gamma_{n}=\operatorname{Gal}\left(k_{\infty} / k_{n}\right)$. Let $A_{n}$ be the $p$-Sylow subgroup of the ideal class group of $k_{n}$ and $D_{n}$ be the subgroup of $A_{n}$ consisting of ideal classes containing

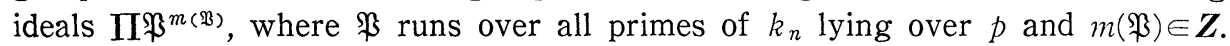
Let $A_{n}^{\prime}$ be the factor group $A_{n} / D_{n}$ (c.f. [6]).

We assume that $k$ is a $C M$ field. Then $k_{\infty}$ is also a $C M$ field. Let $\jmath$ denote the complex conjugation of $k_{\infty}$. For any $\boldsymbol{Z}[\{1, j\}]$-module $M$, let

$$
M^{-}=\{a \in M \mid(1+j) a=0\} .
$$

(0.1) Definition. Let $A_{\infty}^{-}=\varliminf A_{\bar{n}}^{-}$and $A_{\infty}^{\prime-}=\varliminf_{m} A_{n}^{\prime-}$, with respect to the natural maps induced from inclusion maps $k_{n} \rightarrow k_{m}$ for $m \geqq n \geqq 0$.

In [3] Greenberg, and in [2] Ferrero and Greenberg have proved that, if $k$ is abelian over $\boldsymbol{Q}$, then the order of $\left(A_{\infty}^{\prime}\right)^{\Gamma^{\prime}} n$ is finite for any $n \geqq 0$. We shall compute its order by using $p$-adic $L$-functions associated to $k$ when the degree of $k$ over $\boldsymbol{Q}$ is prime to $p$.

In the following, we assume that $k$ is a finite imaginary abelian extension of $\boldsymbol{Q}$ whose degree is prime to $p$. Let $G$ denote the $\operatorname{Galois} \operatorname{group} \operatorname{Gal}(k / \boldsymbol{Q})$ and $\hat{G}$ be its character group $\operatorname{Hom}\left(G, \overline{\boldsymbol{Q}}_{p}^{\times}\right)$, where $\overline{\boldsymbol{Q}}_{p}$ is a fixed algebraic closure of $\boldsymbol{Q}_{p}$, We also consider $\hat{G}$ as the set of primitive Dirichlet characters with values in $\overline{\boldsymbol{Q}}_{p}$ which are associated to the extension $k / \boldsymbol{Q}$ by class field theory. Let $\omega$ be the Teichmüller character module $p$. Take $\phi \in \hat{G}$ with $\phi \neq \omega$ and $\phi(j)$ $=-1$. Let $L_{p}\left(s ; \omega \phi^{-1}\right)$ be the $p$-adic $L$-function attached to $\omega \phi^{-1}$. For $\kappa \in 1+p \boldsymbol{Z}_{p}$ with $\kappa \notin 1+p^{2} \boldsymbol{Z}_{p}$, using Iwasawa's construction of $p$-adic $L$-functions, we have the unique power series $f\left(T ; \omega \phi^{-1}\right) \in \Lambda_{\phi}$ such that

$$
f\left(\kappa^{s-1} ; \omega \phi^{-1}\right)=L_{p}\left(s ; \omega \phi^{-1}\right),
$$

where $\boldsymbol{Z}_{p}[\phi]=\boldsymbol{Z}_{p}[$ all values of $\left.\phi\}\right]$ and $\Lambda_{\phi}=\boldsymbol{Z}_{p}[\phi][[T]]$. We note that

Received February 25, 1982 


$$
f\left(0 ; \omega \phi^{-1}\right)=L_{p}\left(0 ; \omega \phi^{-1}\right)=\left(1-\phi^{-1}(p)\right) L\left(0 ; \phi^{-1}\right) .
$$

(0.2) Definition. We define $\hat{f}\left(T ; \omega \phi^{-1}\right) \in \Lambda_{\phi}$ by

$$
\hat{f}\left(T ; \omega \phi^{-1}\right)= \begin{cases}f\left(T ; \omega \phi^{-1}\right) / T & \text { if } \phi(p)=1, \\ f\left(T ; \omega \phi^{-1}\right) & \text { otherwise. }\end{cases}
$$

Ferrero and Greenberg [2] have proved that $\hat{f}\left(0 ; \omega \phi^{-1}\right) \neq 0$. Then we see that

$$
\hat{f}\left(\zeta-1 ; \omega \phi^{-1}\right) \neq 0 \text { for all } \zeta \text { with } \zeta^{p^{n}}=1 \text { and } n \geqq 0 \text {. }
$$

Hence the order of

$$
\Lambda_{\phi} /\left(\hat{f}\left(T ; \omega \phi^{-1}\right), \omega_{n}\right)
$$

is finite, where $\omega_{n}=(1+T)^{p^{n}}-1$.

For a finite set $A$, let ${ }^{\#} A$ denote the cardinality of $A$. A representation of a group $G$ will be called $\boldsymbol{Q}_{p}$-rrreducible if it is defined over $\boldsymbol{Q}_{p}$ and irreducible over $\boldsymbol{Q}_{p}$. A character of $G$ will be called $\boldsymbol{Q}_{p}$-irreducible if it is the character of some $\boldsymbol{Q}_{p}$-irreducible representation of $G$.

(0.3) THEOREM. Assume that

(1) $k / \boldsymbol{Q}$ is a finite abelian extension,

(2) $k$ is imaginary, and

(3) the degree $[k: Q]$ is prime to $p$.

Then we have

$$
\#\left(A_{\infty}^{\prime-}\right)^{\Gamma_{n}}=\# \bigoplus_{\Phi} \Lambda_{\dot{\phi}} /\left(\hat{f}\left(T ; \omega \dot{\phi}^{-1}\right), \omega_{n}\right) \quad \text { for all } n \geqq 0,
$$

where $\Phi$ runs over all $\boldsymbol{Q}_{p}$-irreducible characters of $G=\operatorname{Gal}(k / \boldsymbol{Q})$ such that $\Phi \neq \omega$, $\Phi(j) \neq \boldsymbol{Q}(1)$ and $\phi$ is an absolutely irreducible component of $\Phi$.

For $a, b \in \boldsymbol{Q}_{p}^{\times}$, we write $\underset{p}{a} b$ if $\operatorname{ord}_{p}(a)=\operatorname{ord}_{p}(b)$. Note that

$$
{ }^{\#} \Lambda_{\phi} /\left(\hat{f}\left(T ; \omega \phi^{-1}\right), \omega_{n}\right) \underset{p}{\sim} \prod_{\psi \zeta p^{n}=1} \prod_{f} \hat{f}\left(\zeta-1 ; \omega \phi^{-1}\right),
$$

where $\phi$ runs over all "conjugates" of $\phi$ over $\boldsymbol{Q}_{p}$.

(0.5) Remark. When no prime of the maximal real subfield $k^{+}$of $k$ lying over $p$ splits in $k$, our formula in Theorem (0.3) is a direct consequence of the analytic class number formula for $k$ (c.f. [1]). But, if there exist some primes of $k^{+}$lying over $p$ which split in $k$, then $\left(A_{\infty}^{-}\right)^{\Gamma}$ is an infinite group and $f\left(0 ; \omega \phi^{-1}\right)$ vanishes for some $\phi$.

(0.6) Remark. To prove Theorem (0.3), we use essentially Gauss sums, GrossKoblitz formula concerning a relation between Gauss sums and special values of Morita's $p$-adic $\Gamma$-function in [4], and Ferrero-Greenberg formula concerning 
$L_{p}^{\prime}(0 ; \chi)$ in $[2]$.

(0.7) Remark. The assumption (3) is not essential. In fact, to prove Theorem (2.1), we need not assume that the degree of $k$ over $\boldsymbol{Q}$ is prime to $p$.

(0.8) Remark. In [2], Ferrero and Greenberg proved Theorem (0.3) when $k$ is imaginary quadratic and $n=0$.

We define fundamental Iwasawa's modules in $\S 1$. In $\S 2$, we reduce Theorem (0.3) to Theorem (2.1). In $\S 3$, we introduce an essential exact sequences of Iwasawa's modules following [3]. And in $\$ 4$, $p$-adic regurators are defined. In $\S 5$, following [4], we define Gauss sums, which we use to combine orders of two modules in Theorem (2.1). And the group of Gauss sums is introduced. In $\S 6$, we prove Theorem (2.1). In $\S 7$, some examples are given.

The author had studied some parts of the content of this paper from December 1980 till July 1981 with H. Nakazato. The author expresses his gratitude to Mr. H. Nakazato for his allowance of publishing this paper.

\section{Notations.}

As usual, $\boldsymbol{Z}, \boldsymbol{Q}, \boldsymbol{R}$, and $\boldsymbol{C}$ denote the ring of rational integers, the field of rational numbers, the field of real numbers, and the field of complex numbers, respectively. For a prime number $p, \boldsymbol{Z}_{p}$ and $\boldsymbol{Q}_{p}$ denote the ring of $p$-adic integers and the field of $p$-adic numbers, respectively. Let $\overline{\boldsymbol{Q}}$ (resp. $\overline{\boldsymbol{Q}}_{p}$ ) be an fixed algebraic closures of $\boldsymbol{Q}$ (resp. $\boldsymbol{Q}_{p}$ ). We also fix embeddings $\overline{\boldsymbol{Q}}{ }_{\hookrightarrow} \boldsymbol{C}$ and $\rho: \overline{\boldsymbol{Q}} \subset \overline{\boldsymbol{Q}}_{p}$.

\section{$\S 1$. Iwasawa's modules.}

Let $k / \boldsymbol{Q}$ be as in Theorem (0.3). Since the degree of $k$ over $\boldsymbol{Q}$ is prime to $p$, all primes of $k$ lying over $p$ are totally ramified in $k$. Since $\left(A_{\infty}^{\prime-}\right)^{\Gamma_{n}}$ is finite for $n \geqq 0$, and the natural maps $A_{n}^{\prime-} \rightarrow A_{m}^{\prime-}$ are injective for $m \geqq n \geqq 0$, we have

(1.1) LEMmA. For any integer $n \geqq 0$, there exists an integer $m_{0}$ such that $\left(A_{\infty}^{\prime-}\right)^{\Gamma_{n}}$ $=\left(A_{m}^{\prime-}\right)^{\Gamma n}$ for all $m \geqq m_{0}$.

Let $Z$ be the decomposition group of $p$ for $k / Q$. Recall that $G=\operatorname{Gal}(k / \boldsymbol{Q})$ and $\hat{G}=\operatorname{Hom}\left(G, \overline{\boldsymbol{Q}}_{p}^{\times}\right)$. For any $\phi \in \hat{G}$, $\operatorname{Tr} \phi$ denotes the $\boldsymbol{Q}_{p}$-irreducible character of $G$ which contains $\phi$ as an absolutely irreducible component, and $e(\operatorname{Tr} \phi)$ denotes the orthogonal idempotent in $\boldsymbol{Z}_{p}[G]$ associated to $\operatorname{Tr} \phi$. We consider $A_{n}, D_{n}$, and $A_{n}^{\prime}$ as $\boldsymbol{Z}_{p}[G]$-modules in the natural way. Since all primes of $k$ lying over $p$ are totally ramified in $k_{n}$, we have

(1.2.) LEMMA. If the restriction $\phi \mid Z$ is not trivial, then $e(\operatorname{Tr} \phi) D_{n}=0$ for all $n \geqq 0$.

Following [3], we have 
(1.3) Lemma. For $m \geqq n \geqq 0$, we have

$$
D_{m}^{-} / D_{n}^{-} \cong\left(\left(\boldsymbol{Z}_{p} / p^{m-n} \boldsymbol{Z}_{p}\right)[G / Z]\right)^{-} \quad \text { as } \boldsymbol{Z}_{p}[G] \text {-modules. }
$$

(1.4) LEMMA. For $m \geqq n \geqq 0$,

$$
0 \longrightarrow D_{n}^{-} \longrightarrow A_{n}^{-} \stackrel{\alpha}{\longrightarrow}\left(A_{m}^{-}\right)^{\Gamma_{n}} / D_{m}^{-} \longrightarrow 0
$$

is an exact sequence of $\boldsymbol{Z}_{p}[G]$-modules, where $\alpha$ is induced by the canonical inclusion $A_{\bar{n}}^{-} \rightarrow A_{m}^{-}$.

For $m \geqq n \geqq 0$, put

$$
M_{n}^{(m)}=\left\{a \in A_{m}^{-} \mid(s-1) a \in D_{m}^{-}\right\},
$$

where $s$ is a generator of $\operatorname{Gal}\left(k_{m} / k_{n}\right)$ (c.f. [3]). Define a homomorphism $\beta: M_{n}^{(m)}$ $\rightarrow D_{m}^{-}$by $\beta(a)=(s-1) a$. Then $D_{m}^{-} \subset \operatorname{Ker} \beta=\left(A_{m}^{-}\right)^{\Gamma_{n}}$. We have an exact sequence of $Z_{p}[G]$-modules:

$$
0 \longrightarrow\left(A_{m}^{-}\right)^{\Gamma_{n}} / D_{m}^{-} \longrightarrow M_{n}^{(m)} / D_{m}^{-} \longrightarrow D_{m}^{-} .
$$

From Lemma (1.4) and since $M_{n}^{(m)} / D_{m}^{-}=\left(A_{m}^{\prime}\right)^{\Gamma_{n}}$, we have an exact sequence of $Z_{p}[G]$-modules :

$$
0 \longrightarrow A_{n}^{\prime-} \longrightarrow\left(A_{m}^{\prime-}\right)^{\Gamma} \longrightarrow D_{m}^{-}
$$

\section{$\S 2$. Reduction.}

In this section, we reduce Theorem (0.3) to the following theorem.

(2.1) THEOREM. Suppose that

(1) $k / \boldsymbol{Q}$ is a finite abelian extension,

(2) $k$ is imaginary, and

(3) $p$ is totally decomposed in $k / \boldsymbol{Q}$.

Then we have

$$
\#\left(A_{\infty}^{\prime-}\right)^{\Gamma} n=\# \bigoplus_{\Phi} \Lambda_{\phi} /\left(\hat{f}\left(T ; \omega \phi^{-1}\right), \omega_{n}\right) \quad \text { for } n \geqq 0,
$$

where $\Phi$ and $\phi$ are as in Theorem (0.3).

In Theorem (2.1), we need not assume that the degree $[k: Q]$ is prime to $p$, and it is essential that $p$ is totally decomposed in $k / \boldsymbol{Q}$.

Let $k / \boldsymbol{Q}$ satisfy the conditions (1), (2), and (3) in Theorem (0.3). For $n \geqq 0$, let $\boldsymbol{Q}_{n}$ be the $n$-th layer of the unique $\boldsymbol{Z}_{p}$-extension of $\boldsymbol{Q}$ (c.f. [6]). Since [k:Q] is prime to $p$, we see that $k_{n}=k \boldsymbol{Q}_{n}$.

(2.2) LEMMA.

$$
\# A_{n}^{-} \underset{p}{\sim} \prod_{\phi} \prod_{\eta} L\left(0 ; \phi^{-1} \eta^{-1}\right) \quad \text { for } n \geqq 0 \text {, }
$$


where $\phi$ rnns over all characters of $\operatorname{Gal}(k / Q)$ such that $\phi(j)=-1$ and $\phi \neq \omega$, and $\eta$ runs over all characters of $\operatorname{Gal}\left(\boldsymbol{Q}_{n} / \boldsymbol{Q}\right)$.

As in $\S 1$, let $Z$ be the decomposition group of $p$ for $k / Q$. Let $X_{1}$ (resp. $X_{2}$ ) be the set of all $\boldsymbol{Q}_{p}$-irreducible characters $\operatorname{Tr} \phi$ of $G=\operatorname{Gal}(k / \boldsymbol{Q})$ such that $\phi(j)$ $=-1$ and $\phi(p)=1$ (resp. $\phi(p) \neq 1$ ). Let $m \geqq n \geqq 0$. Put $A_{m, 2}^{\prime-}=\underset{\phi \in X_{\imath}}{\bigoplus} e(\Phi) A_{m}^{\prime-}$, and $A_{m, i}^{-}=\underset{\phi \in X_{i}}{\bigoplus_{1}} e(\Phi) A_{m}^{-}$for $i=1$ and 2. Then $A_{m}^{\prime-}=A_{m, 1}^{\prime-} \oplus A_{m, 2}^{\prime-}$, and $A_{m}^{-}=A_{m, 1}^{-} \oplus A_{m, 2}^{-}$. From Lemma (1.2) and (1.6), we see that

$$
\left(A_{m, 2}^{\prime-}\right)^{\Gamma_{n}}=A_{n, 2}^{\prime-}=A_{n, 2}^{-} .
$$

Let $A\left(k^{Z}\right)_{n}$ denote the $p$-Sylow subgroup of the ideal class group of $\left(k^{Z}\right)_{n}$, where $\left(k^{Z}\right)_{n}$ is the $n$-th layer of the cyclotomic $\boldsymbol{Z}_{p}$-extension of the fixed field $k^{Z}$ of $Z$. Then

$$
A\left(k^{Z}\right)_{n}^{-} \cong A_{n, 1}^{-} \text {and } A\left(k^{Z}\right)_{m}^{\prime-} \cong A_{m, 1}^{\prime-} .
$$

By Lemma (2.2) for $k^{z}$, we have

$$
\# A\left(k^{Z}\right)_{n}^{-} \underset{p}{\sim} \prod_{\phi} \prod_{\eta} L\left(0 ; \phi^{-1} \eta^{-1}\right),
$$

where $\phi$ runs over all characters of $\operatorname{Gal}(k / \boldsymbol{Q})$ such that $\phi(j)=-1, \phi \neq \omega$, and $\phi \mid Z=1$, and $\eta$ runs over all characters of $\operatorname{Gal}\left(\boldsymbol{Q}_{n} / \boldsymbol{Q}\right)$.

In the rest of this section, we shall prove the following lemma.

(2.6) LEMMA. Theorem (0.3) follows from Theorem (2.1).

Proof. Assume that $k$ satisfies the conditions (1), (2), and (3) in Theorem (0.3). For any $n \geqq 0$, there exists an integer $m \geqq n$ such that

$$
\left(A\left(k^{Z}\right)_{\infty}^{\prime-}\right)^{\Gamma_{n}}=\left(A\left(k^{Z}\right)_{m}^{\prime-}\right)^{\Gamma_{n}} \text { and }\left(A_{\infty}^{\prime-}\right)^{\Gamma_{n}}=\left(A_{m}^{\prime-}\right)^{\Gamma_{n}} .
$$

Hence, by (2.3) and (2.4), we have

$$
\left(A_{\infty}^{\prime-}\right)^{\Gamma_{n}}=\left(A_{m, 1}^{\prime-}\right)^{\Gamma_{n}} \oplus\left(A_{m, 2}^{\prime-}\right)^{\Gamma_{n}} \cong\left(A\left(k^{Z}\right)_{\infty}^{\prime-}\right)^{\Gamma_{n}} \oplus A_{\bar{n}, 2}^{-} .
$$

By Theorem (2.1) for $k^{z}$, we have

$$
\begin{aligned}
\#\left(A\left(k^{Z}\right)_{\infty}^{\prime-}\right)^{\Gamma_{n}} & =\bigoplus_{\Psi}^{\#} \Lambda_{\phi} /\left(\hat{f}\left(T ; \omega \phi^{-1}\right), \omega_{n}\right) \\
& =\underset{\phi \in X_{1}}{\#} \Lambda_{\phi} /\left(\hat{f}\left(T ; \omega \phi^{-1}\right), \omega_{n}\right),
\end{aligned}
$$

where $\Psi$ and $\psi$ are as in Theorem (2.1) with respect to $k^{z}$. On the other hand, since $A_{n}^{-}=A_{\bar{n}, 1}^{-} \oplus A_{n, 2}^{-} \cong A\left(k^{Z}\right)_{n}^{-} \oplus A_{n, 2}^{-}$, from (2.2) and (2.5), we see that

$$
{ }^{\#} A_{\bar{n}, 2}^{-} \underset{p}{\sim} \prod_{\phi \mid Z \neq 1} \prod_{\eta} L\left(0 ; \phi^{-1} \eta^{-1}\right) \underset{p}{\sim} \prod_{\phi \mid Z \neq 1} \prod_{\zeta{ }^{n}=1} \hat{f}\left(\zeta-1 ; \omega \phi^{-1}\right) .
$$

Combining (2.7), (2.8), and (2.9), we obtain Lemma (2.6). 


\section{$\S 3$ The group of imaginary $p$-units.}

In the following, we assume that $k$ satisfies the conditions (1)-(3) in Theorem (2.1). For $n \geqq 0$, let $H_{n}$ be the group of $p$-units of $k_{n}$ :

$$
H_{n}=\left\{\alpha \in k_{n}^{\times} \mid(\alpha)=\text { product of primes of } k_{n} \text { lying over } p\right\} \text {. }
$$

Let $m \geqq n \geqq 0$. Let $N_{m, n}: k_{m} \rightarrow k_{n}$ be the norm map. Recall that

$$
M_{n}^{(m)}=\left\{a \in A_{m}^{-} \mid(s-1) a \in D_{m}^{-}\right\},
$$

where $s$ is a generator of $\operatorname{Gal}\left(k_{m} / k_{n}\right)$.

(3.1) Definition. We define a homomorphism

$$
\varphi_{n}^{(m)}: M_{n}^{(m)} \longrightarrow H_{n}^{1-\jmath} / N_{m, n}\left(H_{m}^{1-j}\right)
$$

in the following way (c.f. $[1,3]$ ). Let $c \in M_{n}^{(m)}$ and let $\mathfrak{A} \in c$. Then $\mathfrak{A}^{1-s}=(\alpha) \mathfrak{B}$ for some $\alpha \in k_{m}^{\times}$and some ideal $\mathfrak{B}$ which is a product of primes of $k_{m}$ lying over $p$. Define

$$
\varphi_{n}^{(m)}(c)=N_{m, n}\left(\alpha^{1-j}\right) \bmod N_{m, n}\left(H_{m}^{1-\jmath}\right) .
$$

This is well-defined (c.f. [3]), and we have

(3.2) LEMMA ([3]). (1) $\operatorname{Ker} \varphi_{n}^{(m)}=\left(A_{m}^{-}\right)^{\Gamma^{\prime} n}$, and

(2) $\operatorname{Im} \varphi_{n}^{(m)}=\left(H_{n}^{1-J} \cap N_{m, n}\left(k_{m}^{\times}\right)^{1-j}\right) / N_{m, n}\left(H_{m}^{1-j}\right)$.

Proof. (1) See [3],

(2) By definition of $\varphi_{n}^{(m)}, \operatorname{Im} \varphi_{n}^{(m)} \subset\left(H_{n}^{1-\jmath} \cap N_{m, n}\left(k_{m}^{\times}\right)^{1-j}\right) / N_{m, n}\left(H_{m}^{1-j}\right)$. Take any $\alpha \in k_{m}^{\times}$such that $N_{m, n}(\alpha) \in H_{n}^{1-\jmath}$. Then $\left(N_{m, n}(\alpha)\right)$ is an ideal of $k_{n}$ which is a product of primes of $k_{n}$ lying over $p$. Since each prime of $k_{n}$ lying over $p$ is totally ramified in $k_{m} / k_{n}$, there exists an ideal $\mathfrak{B}$ of $k_{m}$ which is a product of primes of $k_{m}$ lying over $p$ such that $\left(N_{m, n}(\alpha)\right)=\mathrm{N}_{m, n}(\mathfrak{B})$. Then $N_{m, n}\left(\alpha \mathfrak{B}^{-1}\right)=(1)$. Thus there exists an ideal $\mathfrak{A}$ of $k_{m}$ such that $(\alpha) \mathfrak{B}^{-1}=\mathfrak{U}^{1-s}$. Let $r$ be an integer prime to $p$ such that the class of $\mathfrak{U}^{r(1-j)}$ is contained in $A_{m}^{-}$. Put $a=$ class of $\mathfrak{Y}^{r(1-j)}$. Then $a \in M_{n}^{(m)}$ and $\varphi_{n}^{(m)}(a)=N_{m, n}\left(\alpha^{2 r(1-j)}\right) \bmod N_{m, n}\left(H_{m}^{1-j}\right)$. Since $\left(H_{n}^{1-\jmath} \cap N_{m, n}\left(k_{m}^{\times}\right)^{1-j}\right) / N_{m, n}\left(H_{m}^{1-j}\right)$ is a finite abelian $p$-group, $\operatorname{Im} \varphi_{n}^{(m)}=\left(H_{n}^{1-\jmath} \cap N_{m, n}\right.$ $\left.\left(k_{m}^{\times}\right)^{1-j}\right) / N_{m, n}\left(H_{m}^{1-j}\right)$.

(3.3) COROLLARY.

$$
0 \longrightarrow A_{n}^{\prime-} \longrightarrow\left(A_{m}^{\prime-}\right)^{\Gamma_{n}} \stackrel{\tilde{\varphi}}{\longrightarrow} \operatorname{Im} \varphi_{n}^{(m)} \longrightarrow 0
$$

is an exact sequence of $\boldsymbol{Z}_{p}[G]$-modules, where $\tilde{\varphi}$ is induced from $\varphi_{n}^{(m)}$ since $\varphi_{n}^{(m)}\left(D_{m}^{-}\right)=0$ (c.f. (1.5) and (1.6)). 


\section{$\S 4$. $p$-adic regurators.}

In this section, we shall define $p$-adic regurators for certain subgroups of $H_{0}^{1-3}$. Assume that $k$ satisfies the conditions (1)-(3) in Theorem (2.1). For $n \geqq 0$, let $E_{n}$ be the unit group of $k_{n}$ and let $\mathbf{P}_{n}$ be the subgroup $\left\{(\alpha) \mid \alpha \in H_{n}\right\}$ of the ideal group of $k_{n}$. From a natural exact sequence $0 \rightarrow E_{n} \rightarrow H_{n} \rightarrow \mathbf{P}_{n} \rightarrow 0$, we have an exact sequence $0 \rightarrow E_{n} \cap H_{n}^{1-\jmath} \rightarrow H_{n}^{1-\jmath} \rightarrow \mathbf{P}_{n}^{1-\jmath} \rightarrow 0$. Let $\mu\left(k_{n}\right)$ denote the group of all roots of unity in $k_{n}$, then we have $E_{n} \cap H_{n}^{1-\jmath}=\mu\left(k_{n}\right) \cap H_{n}^{1-\jmath}$. Hence, we have (letting $n=0$ )

$$
\mu(k) H_{0}^{1-\jmath} / \mu(k) \cong \mathbf{P}_{0}^{1-\jmath} .
$$

We note that $\mathbf{P}_{0}^{1-\jmath}$ is a free $\boldsymbol{Z}$-module of $\operatorname{rank} g=[k: \boldsymbol{Q}] / 2$. Assume that $M$ is a submodule of $\mu(k) H_{0}^{1-\jmath}$ such that $\mu(k) M / \mu(k)$ has rank $g$. Let $m_{1}, m_{2}, \cdots, m_{g}$ be a system of elements of $\mu(k) M$ such that $m_{1} \bmod \mu(k), \cdots, m_{g} \bmod \mu(k)$ are $Z$-basis of $\mu(k) M / \mu(k)$. Let $s_{1}, s_{2}, \cdots, s_{g}$ be a system of representatives of $G /\{1, j\}$. Let $\log _{p}$ denote the $p$-adic logarithm from $\boldsymbol{Q}_{p}^{\times}$into $\boldsymbol{Q}_{p}$ normalized by $\log _{p} p=0$ and $\log _{p} \zeta=0$ for $\zeta^{p-1}=1$ (c.f. [5]). Recall that $\rho: \overline{\mathbf{Q}}_{\hookrightarrow} \overline{\mathbf{Q}}_{p}$ is the fixed embedding. Then $\rho(k) \subset \boldsymbol{Q}_{p}$ by the assumption (3) in Theorem (2.1).

Definition. We define the $p$-adic regurator of $M$ by

$$
R_{p}(M)=\operatorname{det}\left(\begin{array}{c}
\log _{p} \rho\left(s_{1} m_{1}\right), \cdots, \log _{p} \rho\left(s_{1} m_{g}\right) \\
\vdots \\
\log _{p} \rho\left(s_{g} m_{1}\right), \cdots, \log _{p} \rho\left(s_{g} m_{g}\right)
\end{array}\right) \quad \text { up to } \pm 1 .
$$

This definition is independent of the choices of $\left(s_{1}, \cdots, s_{g}\right)$ and $\left(m_{1}, \cdots, m_{g}\right)$.

(4.3) Lemma. Let $M_{1} \subset M_{2}$ be submodules of $\mu(k) H_{0}^{1-j}$ such that $R_{p}\left(M_{1}\right) \neq 0$. Then, $R_{p}\left(M_{2}\right) \neq 0$, and

$$
\frac{R_{p}\left(M_{1}\right)}{R_{p}\left(M_{2}\right)}=\left(\mu(k) M_{2}: \mu(k) M_{1}\right) \quad \text { up to } \pm 1
$$

\section{$\S 5$. Gauss sums.}

In this section, we recall Gauss sums in [4]. Assume that $k$ satisfies the conditions (1)-(3) in Theorem (2.1). Let $N$ be the conductor of $k / Q$. Since $p$ is totally decomposed in $k, N$ is prime to $p$. Let $K=\boldsymbol{Q}\left(\mu_{N}\right), G_{N}=\operatorname{Gal}(K / \boldsymbol{Q})$, $H=\mathrm{Gal}(K / k)$, and let $D$ be the decomposition group of $p$ for $K / \boldsymbol{Q}$, where $\mu_{N}$ denote the group of all $N$-th roots of unity in $\overline{\boldsymbol{Q}}$. For any $t \in \boldsymbol{Z}$ such that $(t, N)=1$, define $s_{t} \in G_{N}$ by $s_{t}(\zeta)=\zeta^{t}$ for all $\zeta \in \mu_{N}$. Then $D=\left\langle s_{p}\right\rangle$. Let $v$ be the place of $\overline{\mathbf{Q}}$ corresponding to the fixed enbedding $\rho: \overline{\mathbf{Q}} \subset \overline{\mathbf{Q}}_{p}$. Let $\mathfrak{p}$ (resp. $\mathfrak{p}_{N}$ ) be the prime of $k$ (resp. $K$ ) which is the restriction of $v$ to $k$ (resp. $K$ ).

Definition. Put 


$$
\mathcal{A}_{N}=\frac{1}{N} \boldsymbol{Z} / \boldsymbol{Z}-\{0 \bmod \boldsymbol{Z}\} \text { and } \mathbf{A}_{N}=\operatorname{Map}\left(\mathcal{A}_{N}, \boldsymbol{Z}\right) .
$$

For $a=(t / N \bmod \boldsymbol{Z}) \in \mathcal{A}_{N}$, let $\delta_{t / N}=\delta_{a} \in \mathbf{A}_{N}$ be the map defined by $\delta_{a}(a)=1$ and $\delta_{a}(b)=0$ for $b \in \mathcal{A}_{N}$ with $b \neq a$. The group $G_{N}$ acts on $\mathcal{A}_{N}$ and $\mathbf{A}_{N}$ by

$$
s_{t}\left(t^{\prime} / N \bmod \boldsymbol{Z}\right)=t t^{\prime} / N \bmod \boldsymbol{Z} \quad \text { for } \quad s_{t} \in G_{N}, \quad t^{\prime} / N \bmod \boldsymbol{Z} \in \mathcal{A}_{N}
$$

and

$$
(s \alpha)(a)=\alpha\left(s^{-1} a\right) \quad \text { for } \quad s \in G_{N}, \quad \alpha \in \mathbf{A}_{N}, \quad a \in \mathcal{A}_{N} .
$$

We define the Gauss sum $g\left(\alpha, \mathfrak{p}_{N}, \Psi \circ \mathrm{Tr}\right)=g\left(\alpha, \mathfrak{p}_{N}\right)$ as in (1.3) and (1.4) of [4].

(5.2) Note. Let $\alpha \in N \mathbf{A}_{N}$. Then $g\left(\alpha, \mathfrak{p}_{N}\right)$ is contained in $K^{D}$. The action of $G_{N}$ on $g\left(\alpha, \mathfrak{p}_{N}\right)$ is given by

$$
g\left(\alpha, \mathfrak{p}_{N}\right)^{s}=g\left(s \alpha, \mathfrak{p}_{N}\right) \quad \text { for } \quad s \in G_{N} .
$$

For $x \in \boldsymbol{R}$, let $\langle x\rangle$ be the unique real number such that $0 \leqq\langle x\rangle\langle 1$ and $x-\langle x\rangle \in \boldsymbol{Z}$. For $a=(t / N \bmod \boldsymbol{Z}) \in \mathcal{A}_{N}$, let $\langle a\rangle=\langle t / N\rangle$, and for $\alpha \in \mathbf{A}_{N}$, let $n(\alpha)$ $=\sum_{a \in \mathcal{A}_{N}} \alpha(a)\langle a\rangle$.

Let $\Gamma_{p}$ be the $p$-adic $\Gamma$-function defined by Morita. As in [4], we define $\Gamma_{p}: \mathbf{A}_{N} \rightarrow \boldsymbol{Z}_{p}$ by

$$
\Gamma_{p}(\alpha)=\prod_{a \in \mathfrak{U}_{N}} \Gamma_{p}(\langle a\rangle)^{\alpha(a)} \quad \text { for } \quad \alpha \in \mathbf{A}_{N} .
$$

The following theorem was proved by Gross and Koblitz [4].

(5.3) Theorem. If $n(\alpha) \in \boldsymbol{Z}$, then

$$
\rho\left(g\left(\alpha, \mathfrak{p}_{N}\right)\right)=(-p)^{n\left(\sum_{s \in D} s \alpha\right)} \Gamma_{p}\left(\sum_{s \in D} s \alpha\right) \quad \text { in } \quad \boldsymbol{Q}_{p} .
$$

(5.4) Corollary. Let $\alpha \in N \mathbf{A}_{N}$. Then

$$
\log _{p} \rho\left(g\left(\alpha, \mathfrak{p}_{N}\right)\right)=\sum_{s \in D} \log _{p} \Gamma_{p}(s \alpha) .
$$

Let $X^{-}=\{\phi \in \hat{G} \mid \phi(j)=-1\}$. Let $M$ be a divisor of $N$. We put $X_{M}=\left\{\phi \in X^{-} \mid\right.$ conductor of $\phi=M\}$ and $H_{M}=\operatorname{Gal}\left(\boldsymbol{Q}\left(\mu_{N}\right) / \boldsymbol{Q}\left(\mu_{M}\right)\right) \subset G_{N}$. Recall that $2 g=[k: \boldsymbol{Q}]$ $={ }^{\#} G=\#\left(G_{N} / H\right)$. Fix a system of representatives $\left\{s_{1}, \cdots, s_{2 g}\right\}$ of $G_{N} / H\left(s_{i} \in G_{N}\right.$, $1 \leqq i \leqq 2 g)$. For $\phi \in X^{-}$, let

$$
e(\phi)=\frac{1}{\# G} \sum_{s \in G} \phi\left(s^{-1}\right) s \in \overline{\boldsymbol{Q}}_{p}[G]
$$

and

$$
\tilde{e}(\phi)=\frac{1}{{ }^{\#} G} \sum_{\imath=1}^{2 g} \phi\left(\left(s_{\imath} H\right)^{-1}\right) s_{i} \in \overline{\mathbf{Q}}_{p}\left[G_{N}\right] .
$$


A CLASS NUMBER FORMULA OF IWASAWA'S MODULES

Then we see that ${ }^{\#} \sum_{\phi \in X_{\bar{M}}^{-}} e(\phi) \in \boldsymbol{Z}[G]$ and ${ }^{\#} \sum_{\phi \in X_{M}^{-}} \tilde{e}(\phi) \in \boldsymbol{Z}\left[G_{N}\right]$.

(5.5) Definition. Let $\mathbf{A}_{k}$ be the submodule of $N \mathbf{A}_{N}$ generated by

$$
\left\{{ }^{\#} G \sum_{\phi \in X_{\bar{M}}^{-}} e(\phi) N \delta_{1 / M}|M| N_{i}\right\} .
$$

(5.6) Definition. We define the Gauss sum $g_{k}(\alpha)$ of $k$ associated to $\alpha \in N \mathbf{A}_{N}$ by

$$
g_{k}(\alpha)=N_{K} D_{/ k}\left(g\left(\alpha, \mathfrak{p}_{N}\right)\right)
$$

and the group $\mathcal{G}_{k}$ of Gauss sums of $k$ by

$$
\mathcal{G}_{k}=\left\{g_{k}(\alpha) \mid \alpha \in \mathbf{A}_{k}\right\} \text {. }
$$

We define a $\boldsymbol{Z}\left[G_{N}\right]$-homomorphism $S_{k}: N \mathbf{A}_{N} \rightarrow \boldsymbol{Z}[G]$ by

$$
S_{k}(\alpha)=\sum_{s \in G_{N}} n(s \alpha)(s H)^{-1} \quad \text { for } \quad \alpha \in N \mathbf{A}_{N} .
$$

We have Stickelberger relations for $k$.

(5.7) If $\alpha \in N \mathbf{A}_{N}$, then

$$
\left(g_{k}(\alpha)\right)=\mathfrak{p}^{S_{k}(\alpha)} \text { in } k \text {. }
$$

For $n \geqq 0$, let $\mathbf{D}_{n}$ be the subgroup of the ideal group of $k_{n}$ generated by primes of $k_{n}$ lying over $p$, and let $\mathbf{G}_{k}=\left\{\left(g_{k}(\alpha)\right) \mid \alpha \in \mathbf{A}_{k}\right\}$. From (5.7), $\mathbf{G}_{k}$ is a $\boldsymbol{Z}[G]$ submodule of $\mathbf{D}_{0}$.

(5.8) PROPOSITION.

$$
\left(\mathbf{D}_{0}^{1-\jmath}: \mathbf{G}_{k}^{1-\jmath}\right)=(2 g N)^{g} \prod_{M \backslash N}\left({ }^{\#} H_{M}\right)^{\# X_{M}^{-}} \prod_{\phi \in X^{-}} L\left(0 ; \phi^{-1}\right) .
$$

Proof. Since $p$ is totally decomposed in $k$, we have

$$
\mathbf{D}_{0}^{1-\jmath}=(1-j) \boldsymbol{Z}[G] \cdot p \cong(1-j) \boldsymbol{Z}[G] .
$$

We compute the index $\left(\mathbf{D}_{0}^{1-\jmath}: \mathbf{G}_{k}^{1-j}\right)$ in $(1-j) \boldsymbol{Z}[G] \otimes \overline{\boldsymbol{Q}}_{p}=(1-j) \overline{\boldsymbol{Q}}_{p}[G]$ and in $e(\phi) \overline{\boldsymbol{Q}}_{p}[G]=e(\phi) \overline{\boldsymbol{Q}}_{p}$ for $\phi \in X^{-}$, because $(1-j) \overline{\boldsymbol{Q}}_{p}[G]=\bigoplus_{\phi \in X^{-}} e(\phi) \overline{\boldsymbol{Q}}_{p}$. Let $M \mid N$ and let $\phi \in X_{M}^{-}$. For $L \mid N$, we have

$$
\begin{aligned}
e(\phi) S_{k}\left(\# G \sum_{\phi \in X_{\bar{L}}^{-}} \tilde{e}(\phi) N \delta_{1 / L}\right) \\
\quad= \begin{cases}e(\phi)^{\#} G N \sum_{s_{t} \in G_{N}}\langle t / M\rangle \phi^{-1}\left(s_{t} H\right) & \text { if } L=M, \\
0 & \text { if } L \neq M .\end{cases}
\end{aligned}
$$

Futhermore we have

$$
\sum_{s_{t} \in G_{N}}\langle t / M\rangle \phi^{-1}\left(s_{t} H\right)=\# H_{M} \sum_{\bar{s}_{t} \in G_{M}}\langle t / M\rangle \phi_{M}^{-1}\left(\bar{s}_{t} \bar{H}\right),
$$


where $\bar{s}_{t}=s_{t} H_{M}, G_{M}=G_{N} / H_{M}, \bar{H}=H H_{M} / H_{M}$, and $\phi_{M}$ is the character of $G_{M} / \bar{H}$ induced from $\phi$. Hence

$$
e(\phi) S_{k}\left({ }^{\sharp} G \sum_{\phi \in X_{\bar{L}}^{\bar{L}}} \tilde{e}(\psi) N \delta_{1 / L}\right)= \begin{cases}e(\phi)^{\#} G N^{\sharp} H_{M} L\left(0 ; \phi^{-1}\right) & \text { if } \quad L=M, \\ 0 & \text { if } \quad L \neq M .\end{cases}
$$

Since $(1-j) e(\phi)=2 e(\phi)$, we have

$$
\left(\mathbf{D}_{0}^{1-\jmath}: \mathbf{G}_{k}^{1-j}\right)=(2 g N)^{g} \prod_{M \mid N}\left({ }^{\#} H_{M}\right)^{\#} X_{M}^{-} \prod_{\rho \in X^{-}} L\left(0 ; \phi^{-1}\right) .
$$

We shall compute the $p$-adic regurator of $\mathcal{G}_{k}^{1-\jmath}$ by using $p$-adic $L$-functions.

(5.9) THEOREM.

$$
R_{p}\left(\mathcal{G}_{k}^{1-j}\right)=(4 g N)^{g} \prod_{M \backslash N}\left(^{\#} H_{M}\right)^{\# X_{M}^{-}} \prod_{\rho \in X^{-}} L_{p}^{\prime}\left(0 ; \omega \phi^{-1}\right) \quad u p \text { to } \pm 1 .
$$

We recall a result of Ferrero and Greenberg [2].

(5.10) Theorem. Let $M \mid N$ and let $\phi \in X_{M}^{-}$. Then

$$
L_{p}^{\prime}\left(0 ; \omega \phi^{-1}\right)=\sum_{\bar{s}_{t} \in G_{M}} \phi^{-1}\left(\bar{s}_{t}\right) \log _{p} \Gamma_{p}\left(s_{t} \delta_{1 / M}\right) .
$$

By (5.10) and (5.4),

$$
L_{p}^{\prime}\left(0 ; \omega \phi^{-1}\right)=\frac{1}{N^{\#} H_{M}} \sum_{s \in G} \phi^{-1}(s) \log _{p} \rho\left(g_{k}\left(N \delta_{1 / M}\right)^{s}\right) .
$$

Put $g_{k, M}=g_{k}\left(N \delta_{1 / M}\right)$. We have, for $s_{t} \in G_{N}$ with $s_{t} H=s$,

$$
\begin{aligned}
\log _{p} \rho\left(g_{k}\left(N^{\#} G \sum_{\psi \in X_{\bar{M}}^{-}} \tilde{e}(\psi) s_{t} \delta_{1 / M}\right)\right) \\
=\log _{p} \rho\left(g_{k, M}{ }^{\# G \sum_{\psi \in X_{M}^{-}} e(\psi) s}\right)
\end{aligned}
$$

(5.12) Claim. Let $L \mid N$ and let $\phi \in X_{\bar{L}}^{-}$. Then

$$
\begin{aligned}
\sum_{s \in G} \phi^{-1}(s) \log _{p} \rho\left(\left(g_{k, M}{ }^{\#} G \sum_{\psi \in X_{M}^{-}} e(\phi) s\right)\right. \\
=\left\{\begin{array}{lll}
\# G \sum_{s \in G} \phi^{-1}(s) \log _{p} \rho\left(\left(g_{k, M}\right)^{s}\right) & \text { if } & L=M, \\
0 & \text { if } & L \neq M .
\end{array}\right.
\end{aligned}
$$

In fact, computing in $\overline{\boldsymbol{Q}}_{p}[G] \otimes \log _{p} \rho\left(\mathcal{G}_{k}^{1-j}\right)=\overline{\boldsymbol{Q}}_{p}[G]=\sum_{\phi} e(\phi) \overline{\mathbf{Q}}_{p}$, because $\log _{p} \rho\left(\mathcal{G}_{k}^{1-j}\right)$ $\subset \boldsymbol{Q}_{p}$, we have

$$
e(\phi) \sum_{s \in G} s^{-1} \log _{p} \rho\left(\left(g_{k, M}{ }^{\# G} \sum_{\phi \in X_{M}^{-}} e(\phi) s\right)\right.
$$




$$
\begin{aligned}
& =e(\phi)^{\#} G \sum_{\psi \in X_{M}^{-}} e(\phi) \sum_{s \in G} s^{-1} \log _{p} \rho\left(\left(g_{k, M}\right)^{s}\right) \\
& =\left\{\begin{array}{lll}
e(\phi)^{\#} G \sum_{s \in G} \phi^{-1}(s) \log _{p} \rho\left(\left(g_{k, M}\right)^{s}\right) & \text { if } & L=M, \\
0 & \text { if } & L \neq M .
\end{array}\right.
\end{aligned}
$$

Proof of Theorem (5.9)

Define the map $\log _{p}: H_{0}^{1-\jmath} \rightarrow(1-j) \boldsymbol{Q}_{p}[G]$ by

$$
\log _{p}(x)=(1-j) \sum_{\bar{s} \in G /\{1, \jmath\}} \log _{p} \rho\left(x^{s}\right) s^{-1} \quad \text { for } \quad x \in H_{0}^{1-\jmath} .
$$

Since $\log _{p}\left(\mathcal{G}_{k}^{1-j}\right) \subset(1-j) \boldsymbol{Q}_{p}[G]$, we compute $R_{p}\left(\mathcal{G}_{k}^{1-j}\right)$ in $(1-j) \boldsymbol{Q}_{p}[G] \otimes \overline{\boldsymbol{Q}}_{p}=$ $(1-j) \overline{\boldsymbol{Q}}_{p}[G]=\bigoplus_{p \in X^{-}} e(\phi) \overline{\boldsymbol{Q}}_{p}$. Since $\mathcal{G}_{k}^{1-\jmath}$ is generated by

$$
\left(g_{k, M}^{1-j}\right){ }_{G \in X_{L}^{-}}^{\sum_{L}} e^{(\psi)} \quad \text { for all } M|N, L| N,
$$

we have from (5.12)

$$
\begin{aligned}
& \pm R_{p}\left(\mathcal{G}_{k}^{1-j}\right)=\prod_{M \backslash N} \prod_{\phi \in X_{M}^{-}} 2^{\#} G \sum_{s \in G} \phi^{-1}(s) \log _{p} \rho\left(\left(g_{k, M}\right)^{s}\right) \\
& =\left(2^{\#} G\right)^{\#} X^{-} \prod_{M \backslash N} \prod_{\phi \in X_{M}^{-}} N^{\#} H_{M} L_{p}^{\prime}\left(0 ; \omega \phi^{-1}\right) . \quad \text { Q. E. D. }
\end{aligned}
$$

Ferrero and Greenberg [2] have proved that $L_{p}^{\prime}\left(0 ; \omega \phi^{-1}\right) \neq 0$. Then we see that $R_{p}\left(\mathcal{G}_{k}^{1-j}\right) \neq 0$. Hence, by Lemma (4.3),

$$
\left.R_{p}\left(H_{0}^{1-j}\right) \neq 0 \quad \text { (c.f. }[3]\right) .
$$

\section{$\S 6$. Proof of Theorem (2.1).}

In this section, we shall prove Theorem (2.1). Let $n \geqq 0$. Recall that $\mathbf{D}_{n}=$ $\left\{\Pi \mathfrak{P}^{m(\mathfrak{P})}|\mathfrak{P}| p\right.$ in $\left.k_{n}, m(\mathfrak{B}) \in \boldsymbol{Z}\right\}$. Let $\mathscr{P}_{n}$ be the principal ideal group of $k_{n}$. Put $\mathbf{P}_{n}=\mathscr{Q}_{n} \cap \mathbf{D}_{n}$ and $\mathscr{D}_{n}=\mathbf{D}_{n} / \mathbf{P}_{n}$. Then

$$
\left.\left(\mathbf{D}_{0}^{1-\jmath}: \mathbf{P}_{0}^{1-j}\right)=\# \mathscr{D}_{0}^{1-\jmath} \quad \text { (up to a } 2 \text {-factor }\right) \underset{p}{\sim} D_{0}^{-} .
$$

From Lemma (4.3) and (5.13), we have

$$
\pm \frac{R_{p}\left(\mathcal{G}_{k}^{1-j}\right)}{R_{p}\left(H_{0}^{1-j}\right)}=\left(\mathbf{P}_{0}^{1-\jmath}: \mathbf{G}_{k}^{1-j}\right)=\frac{\left(\mathbf{D}_{0}^{1-\jmath}: \mathbf{G}_{k}^{1-j}\right)}{\left(\mathbf{D}_{0}^{1-j}: \mathbf{P}_{0}^{1-j}\right)} \quad \text { (up to a } 2 \text {-factor). }
$$

Thus, from Proposition (5.8) and Theorem (5.9), we have

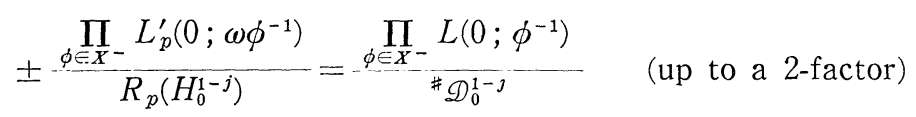

(6.2) Lemma. For $m \geqq 0$, 


$$
\#\left(H_{0}^{1-\jmath} /\left(H_{0}^{1-\jmath} \cap N_{m, 0}\left(k_{m}^{\times}\right)^{1-j}\right)\right) \underset{p}{\sim} \frac{p^{(m+1) g}}{R_{p}\left(H_{0}^{1-j}\right)} .
$$

Proof. Recall the map $\log _{p}$ in $\S 5$. Since $k_{m} / k$ is totally ramified at $\mathfrak{p}$, by Hasse's norm theorem following [3], we have

$$
x \in N_{m, 0}\left(k_{m}^{\times}\right)^{1-\jmath} \Leftrightarrow \log _{p}(x) \in(1-j) p^{m+1} Z_{p}[G], \quad \text { for } \quad x \in H_{0}^{1-\jmath} \text {. Q. E. D. }
$$

(6.3) For $n \geqq 0$ and for a sufficiently large $m \geqq n$,

$$
\boldsymbol{Z}_{p} \otimes\left(H_{0}^{1-\jmath} \cap N_{m, 0}\left(k_{m}^{\times}\right)^{1-j}\right)=\boldsymbol{Z}_{p} \otimes\left(N_{n, 0}\left(H_{n}^{1-\jmath} \cap N_{m, n}\left(k_{m}^{\times}\right)^{1-\jmath}\right) .\right.
$$

In fact, by Lemma (6.2), there exists a sufficiently large $m \geqq n$, such that

$$
\boldsymbol{Z}_{p} \otimes N_{n, 0}\left(H_{n}^{1-\jmath}\right) \subset \boldsymbol{Z}_{p} \otimes\left(H_{0}^{1-\jmath} \cap N_{m, 0}\left(k_{m}^{\times}\right)^{1-j}\right) .
$$

(6.4) For $m \geqq n \geqq 0$, we have

$$
\begin{aligned}
&\left(\boldsymbol{Z}_{p}\right.\left.\otimes H_{0}^{1-\jmath}: \boldsymbol{Z}_{p} \otimes\left(H_{0}^{1-\jmath} \cap N_{m, 0}\left(k_{m}^{\times}\right)^{1-j}\right)\right) \\
& \quad=\left(\boldsymbol{Z}_{p} \otimes \mathbf{P}_{0}^{1-\jmath}: \boldsymbol{Z}_{p} \otimes\left(\mathbf{P}_{0}^{1-\jmath} \cap N_{m, 0}\left(\mathscr{P}_{m}^{1-j}\right)\right)\right)
\end{aligned}
$$

and

$$
\begin{aligned}
\left(\boldsymbol{Z}_{p}\right. & \otimes\left(H_{n}^{1-\jmath} \cap N_{m, n}\left(k_{m}^{\times}\right)^{1-\jmath}: \boldsymbol{Z}_{p} \otimes N_{m, n}\left(H_{m}^{1-j}\right)\right) \\
& =\left(\boldsymbol{Z}_{p} \otimes\left(\mathbf{P}_{n}^{1-\jmath} \cap N_{m, n}\left(\mathcal{Q}_{m}^{1-j}\right)\right): \boldsymbol{Z}_{p} \otimes \mathrm{N}_{m, n}\left(\mathbf{P}_{m}^{1-j}\right)\right) .
\end{aligned}
$$

For any $n \geqq 0$, let $m$ be an integer satisfying (6.3). Since the norm maps $\mathrm{N}_{m, n}: \mathbf{D}_{m}^{1-\jmath} \rightarrow \mathbf{D}_{n}^{1-\jmath}$ and $\mathrm{N}_{n, 0}: \mathbf{D}_{n}^{1-\jmath} \rightarrow \mathbf{D}_{0}^{1-\jmath}$ are bijective, we have the following diagram :

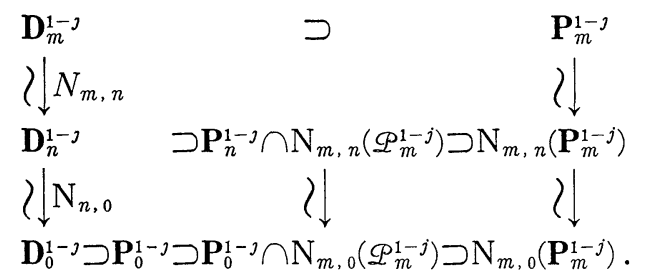

We have, by (6.5),

$$
\begin{aligned}
\left(\boldsymbol{Z}_{p}\right. & \left.\otimes\left(\mathbf{P}_{n}^{1-\jmath} \cap \mathrm{N}_{m, n}\left(\mathscr{P}_{m}^{1-j}\right)\right): \boldsymbol{Z}_{p} \otimes \mathrm{N}_{m, n}\left(\mathbf{P}_{m}^{1-j}\right)\right) \\
\quad= & \frac{\left(\boldsymbol{Z}_{p} \otimes \mathbf{D}_{m}^{1-\jmath}: \boldsymbol{Z}_{p} \otimes \mathbf{P}_{m}^{1-j}\right)}{\left(\boldsymbol{Z}_{p} \otimes \mathbf{D}_{0}^{1-j}: \boldsymbol{Z}_{p} \otimes \mathbf{P}_{0}^{1-j}\right)\left(\boldsymbol{Z}_{p} \otimes \mathbf{P}_{0}^{1-\jmath}: \boldsymbol{Z}_{p} \otimes\left(\mathbf{P}_{0}^{1-\jmath} \cap N_{m, 0}\left(\mathscr{P}_{m}^{1-j}\right)\right)\right)} .
\end{aligned}
$$

Hence, by Lemma (3.2) and (6.4), we have

$$
\# \operatorname{Im} \varphi_{n}^{(m)}=\frac{\#\left(D_{m}^{-} / D_{0}^{-}\right)}{\left(\boldsymbol{Z}_{p} \otimes H_{0}^{1-\jmath}: \boldsymbol{Z}_{p} \otimes\left(H_{0}^{1-j} \cap N_{m, 0}\left(k_{m}^{\times}\right)^{1-j}\right)\right)} .
$$

Thus, by Lemma (6.2) and Lemma (1.3), we have 


$$
{ }^{\#} \operatorname{Im} \varphi_{n}^{(m)} \underset{p}{\sim} p^{-g} R_{p}\left(H_{0}^{1-j}\right) .
$$

Note that

$$
L_{p}^{\prime}\left(0 ; \omega \phi^{-1}\right) \underset{p}{\sim} p \hat{f}\left(0 ; \omega \phi^{-1}\right) .
$$

Proof of Theorem (2.1)

For a given $n$, take an integer $m \geqq n$ such that $\left(A_{\infty}^{\prime-}\right)^{\Gamma_{n}}=\left(A_{m}^{\prime-}\right)^{\Gamma_{n}}$ and (6.3) holds. By using (3.3), (6.1), (6.6) and (6.7), we have

because

$$
\#\left(A_{m}^{-}\right)^{\Gamma_{n}} \sim \prod_{p \in X^{-}} \prod_{\zeta^{p n=1}} \tilde{f}\left(\zeta-1 ; \omega \phi^{-1}\right),
$$

$$
\#\left(A_{n}^{\prime-} / A_{0}^{\prime-}\right) \underset{p}{\sim} \prod_{\phi \in X^{-}} \prod_{\substack{\zeta \neq n=1 \\ \zeta \neq 1}} \tilde{f}\left(\zeta-1 ; \omega \phi^{-1}\right) .
$$

Hence, we complete the proof of Theorem (2.1).

\section{§ 7. Examples.}

1. Let $p=5$, and $k$ be the unique subfield of $\boldsymbol{Q}(\exp (2 \pi i / 1949))$ of degree 4 over $\boldsymbol{Q}$. Then 5 splits completely in $k / \boldsymbol{Q}$. There are two imaginary $\boldsymbol{Q}_{5}$-irreducible characters of $\operatorname{Gal}(k / \boldsymbol{Q})$. We have ${ }^{\#} A_{0}^{-}=5^{3}$ and $D_{0}^{-} \cong \boldsymbol{Z} / 5 \boldsymbol{Z} \oplus \boldsymbol{Z} / 5 \boldsymbol{Z}$, by an easy computation. Hence $\# A_{0}^{\prime-}=5$. By a computation (modulo congruence) of the coefficients of $f\left(T ; \omega \phi^{-1}\right)$, we have

$$
\# \bigoplus_{\phi} \Lambda_{\phi} /\left(\hat{f}\left(T ; \omega \phi^{-1}\right), \omega_{0}\right)=5^{3} .
$$

Using Theorem (0.3) we have \# $\left(A_{\infty}^{\prime-}\right)^{\Gamma_{0}}=5^{3}$. Hence $A_{0}^{\prime-} \subsetneq\left(A_{\infty}^{\prime-}\right)^{\Gamma_{0}}$. Moreover, we see that $A_{1}^{-}=5^{9}, \# D_{1}^{-}=5^{4}$, and $A_{1}^{\prime-}=5^{5}$. Note that $\lambda^{-}$-invariant of $k$ (for $p=5$ ) is 6 .

2. Let $p=5$, and $k$ be the unique subfield of $\boldsymbol{Q}(\exp (2 \pi i / 2269))$ of degree 4 over $\boldsymbol{Q}$. We have

$$
\bigoplus_{\Phi} \Lambda_{\phi} /\left(\hat{f}\left(T ; \omega \phi^{-1}\right), \omega_{0}\right)=\{0\} .
$$

By using Theorem (0.3), we have $\left(A_{\infty}^{\prime-}\right)^{\Gamma_{0}}=\{0\}$. Hence $D_{0}^{-}=\# A_{0}^{-}=5^{3}$. Note that $\lambda^{-}$-invariant of $k$ is 2 . We have $A_{0}^{\prime-}=\left(A_{\infty}^{\prime-}\right)^{\Gamma_{0}}=\{0\}$.

\section{REFERENCES}

[1] Coates, J., Lichtenbaum, S., On $l$-adic zeta functions. Ann. Math. 98 (1973), 498-550.

[2] Ferrero, B., Greenberg, R., On the behavior of $p$-adic $L$-functions at $s=0$. Inv. Math. 50 (1978), 91-102.

[3] Greenberg, R., On a certain $l$-adic representation. Inv. Math. 21 (1973), 117124. 
[4] Gross, B., Koblitz, N., Gauss sums and the p-adic $\Gamma$-function. Ann. Math. 109 (1979), 569-581.

[5] Iwasawa, K., Lectures on p-adic L-functions. Ann. Math. Studies 74, Princeton University Press, 1972.

[6] Iwasawa, K., On $Z_{l}$-extensions of algebraic number fields. Ann. Math. 98 (1973), 246-326.

[7] Federer, L., Gross, B., Regurators and Iwasawa modules. Inv. Math. 62 (1981), 443-457.

[8] Gross, B., $p$-adic $L$-series at $s=0$. (to appear)

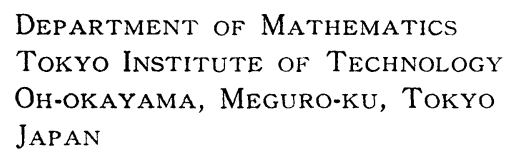

\section{Addendum in proof}

We received from Gross the preprint of [8] after we had sent him the preprint of this paper. There is a partial overlap between the content of [8] and that of this paper. Assuming Conjecture (5.3) of the paper of Federer and Gross [7] and combinig their Proposition (3.9) [7], one will get Theorem (0.3) in this paper in the case of $n=0$. In [7], they announced that Conjecture (5.3) of abelian case was proved in [8]. In this paper, without assuming Conjecture (5.3) [7], we prove Theorem (0.3) for all $n \geqq 0$ by using Theorem (5.9). 